\title{
Oxygen ion reflection at earthward propagating dipolarization fronts in the
}

\section{Magnetotail}

\author{
S. J. Zhao ${ }^{1}$, S. Y. Fu ${ }^{1}$, W. J. Sun ${ }^{2,3}$, G. K. Parks ${ }^{4}$, X. Z. Zhou ${ }^{1}$, Z. Y. Pu ${ }^{1}$, D. \\ $Z_{\text {Zhao }}{ }^{1}$, T. Wu ${ }^{1}$, F. B. Yu ${ }^{1}$, Q. G. Zong ${ }^{1}$ \\ ${ }^{1}$ School of Earth and Space Sciences, Peking University, Beijing 100871, China. \\ ${ }^{2}$ Key Laboratory of Earth and Planetary Physics, Institute of Geology and \\ Geophysics, Chinese Academy of Sciences, Beijing 100029, China. \\ ${ }^{3}$ Department of Climate and Space Sciences and Engineering, University of Michigan, \\ Ann Arbor, Michigan 48109, USA. \\ ${ }^{4}$ Space Sciences Laboratory, University of California, Berkeley, California, USA
}

Corresponding author: S. Y. Fu (suiyanfu@pku.edu.cn)

\section{Key Points:}

- The reflection of oxygen ions at the depolarization front are studied in both observations and simulations.

- A flux dropout could be observed in the $\mathrm{O}^{+}$spectrum, whose contour is energy-dependent and highly correlated with the gyro-motion of different energy $\mathrm{O}^{+}$.

This is the author manuscript accepted for publication and has undergone full peer review but has not been through the copyediting, typesetting, pagination and proofreading process, which may lead to differences between this version and the Version of Record. Please cite this article as doi: 10.1029/2018JA025689

This article is protected by copyright. All rights reserved. 
- $\mathrm{O}^{+}$can extend to a further position after the DF due to its larger gyro-radius than that of $\mathrm{H}^{+}$with the same energy.

\begin{abstract}
A dipolarization front (DF) is known as the leading edge of an earthward high-speed flow with a sharp enhancement in the northward magnetic field $\left(B_{\mathrm{z}}\right)$. Analysis of an event observed by Cluster shows that the behavior of oxygen ions $\left(\mathrm{O}^{+}\right)$around the DF is very different from protons $\left(\mathrm{H}^{+}\right)$. After the crossing of the $\mathrm{DF}$, the $\mathrm{O}^{+}$density decreases more gradually than $\mathrm{H}^{+}$. The distance between the density minimum of $\mathrm{O}^{+}$ and the DF layer is $\sim 4$ times longer than that of $\mathrm{H}^{+}$, which is close to their gyro-radii ratio with the same energy. A flux dropout is observed in the $\mathrm{O}^{+}$energy spectrum, whose energy dependence indicates that ions with higher energies can reach locations farther tailward of the DF. Similar variations are also seen in studies of 22 events in which a common pattern of ion properties is obtained by performing a superposed epoch analysis. Finally, using backward tracing test-particle simulations, we reproduce the characteristics of the flux dropout and verify that the time dependence of the dropout is highly correlated with the gyro-motion of different energy $\mathrm{O}^{+}$behind the DF. All these results provide a further understanding of ion dynamics associated with DFs and suggest that the observed $\mathrm{O}^{+}$ions are reflected within a half gyro-motion in the central plasma sheet.
\end{abstract}


Keywords: Oxygen Ions, Dipolarization Front, Flux Dropout, Reflection Process

\section{Introduction}

Dipolarization fronts (DFs), often considered as the leading edges of earthward propagating plasma flows, are magnetic structures with sharp enhancements in the northward magnetic field $\left(B_{\mathrm{z}}\right)$ in the magnetotail [e.g., Nakamura et al., 2002; Runov et al., 2009; Fu et al., 2011]. DFs can be interpreted as current layers that separate the hot tenuous particle population in plasma flows from the cold dense population in the ambient plasma sheet [e.g., Sergeev et al., 2009; Runov et al., 2011; Sun et al., 2014a]. These current layers have thicknesses estimated to be comparable to plasma sheet proton gyro-radius ( $500 \mathrm{~km}$ to $\sim 1000 \mathrm{~km}$ ) [e.g., Runov et al., 2009; Liu et al., 2013; Sun et al., 2014b]. They are also associated with kinetic features [e.g., Sergeev et al., 2009; Fu et al., 2012; Sun et al., 2014b] and field-aligned currents [e.g., Liu et al., 2013, 2015; Sun et al., 2013, 2014a] as well.

In the earthward propagation of DFs, precursor signatures that include gradual enhancements in plasma density, velocity, and pressure are commonly detected $\sim 20$ to $\sim 60 \mathrm{~s}$ prior to the sharp enhancement of $B_{z}$ [e.g., Ohtani et al., 2004; Zhou et al., 2010, 2011; Zhao et al., 2016]. These signatures have been interpreted as pre-existing plasma sheet particles being accelerated and reflected by the approaching DF [Zhou et al., 2010, 2011; Wu and Shay, 2012; Eastwood et al., 2015]. Test particle [e.g., Zhou et al., 2010, 2011] as well as Particle-In-Cell (PIC) [Eastwood et al., 2015] simulations have revealed that the pre-existing plasma sheet ions can be reflected by performing a half gyro-motion and be accelerated by the dawn-dusk electric field carried by the DF. In addition to these precursor signatures, $B_{z}$ can often decrease 
ahead of the DF (referred to as $B_{\mathrm{z}}$ dips). The $B_{\mathrm{z}}$ dips could be a remnant feature of an earthward-traveling flux rope, which is characterized by a helical magnetic configuration [e.g., Slavin et al., 2003]. And in recent studies, it has also been suggested that this feature is associated with a dawnward current inside the dip region [Yao et al., 2013, 2015; Zhou et al., 2014; Lu et al., 2016]. Pan et al. [2015] proposed that the $B_{\mathrm{Z}}$ dip could have resulted from the dawnward diamagnetic currents formed by the DF-reflected ions. All of the features mentioned above can be understood in the framework of a reflection process, but there have been no direct observations of the half gyro-motion during the reflection. Moreover, protons $\left(\mathrm{H}^{+}\right)$have been assumed to be the only ion species in all the above studies. How the heavy ions (e.g. $\mathrm{O}^{+}$) behave have not been investigated.

It is known that oxygen ions $\left(\mathrm{O}^{+}\right)$originating from the ionosphere could play an important role in the Earth's magnetospheric dynamics [e.g., Fu et al., 2001; Seki et al., 2001; Shay and Swisdak, 2004]. In the plasma sheet, the $\mathrm{O}^{+}$density is normally smaller than $\mathrm{H}^{+}$density, and the motions of $\mathrm{O}^{+}$ions are decoupled from $\mathrm{H}^{+}$ions [e.g., Kistler et al., 2005; Zelenyi et al., 2006; Wu et al., 2016; Nilsson et al., 2016]. For example, in bursty bulk flows (BBFs), $\mathrm{O}^{+}$move predominantly duskward while the $\mathrm{H}^{+}$mainly drift earthward [Kistler et al., 2005]. Moreover, noting also that the $\mathrm{O}^{+}$has a larger gyro-radius, it is unlikely that $\mathrm{O}^{+}$will be transported and accelerated in the BBFs the same as protons, which are of limited spatial and temporal extent [Nilsson et al., 2016].

In a recent test particle simulation, Greco et al. [2015] studied the acceleration of heavy ions (including $\mathrm{O}^{+}$and $\mathrm{Na}^{+}$) associated with DFs and found that when the ion species are initially cold, heavy ions could gain more energy than $\mathrm{H}^{+}$at local DFs, 
depending on their initial gyro-radii. Liang et al. [2017] investigated the acceleration of $\mathrm{O}^{+}$with 2.5-D implicit PIC simulation and showed multiple peaks in the $\mathrm{O}^{+}$ distribution function ahead of the DF coming from the lobe and pre-existing $\mathrm{O}^{+}$ions. According to their results, the lobe oxygen ions can be accelerated by the Hall electric field and enter the downstream outflow region; the pre-existing current sheet $\mathrm{O}^{+}$ions are reflected by the earthward propagating DF and form a reflected beam.

Since the gyro-radius of $\mathrm{O}^{+}$is four times larger than that of $\mathrm{H}^{+}$with the same energy, the response to the DF is expected to be different. This has motivated us to investigate the $\mathrm{O}^{+}$features around the DFs. In this study, we will investigate the variations of $\mathrm{O}^{+}$ properties (i.e., density, energy flux) around DFs and compare $\mathrm{O}^{+}$features with that of $\mathrm{H}^{+}$using both observations and test particle simulations. We first present a case study to analyze the $\mathrm{O}^{+}$flux dropout associated with a $\mathrm{DF}$, in which the dropout boundary is observed to be energy dependent. Accordingly, we study the $\mathrm{O}^{+}$reflection process and discuss the distinct signatures between $\mathrm{O}^{+}$and $\mathrm{H}^{+}$. We then expand our study to multiple events and show that backward tracing test-particle Liouville simulations can reproduce the characteristics of $\mathrm{O}^{+}$and $\mathrm{H}^{+}$around DFs.

\section{Observations}

This study employs the data from Cluster [Escoubet et al., 2001]. The magnetic field data are obtained from the fluxgate magnetometer (FGM) [Balogh et al., 2001] with full-time resolution $(\sim 22.5 \mathrm{~Hz})$. The ion data are provided by the ion composition and distribution function analyzer (CODIF) which is part of the ion spectrometry (CIS) experiment [Rème et al., 2001]. CODIF can resolve $\mathrm{H}^{+}, \mathrm{He}^{2+}, \mathrm{He}^{+}$, and $\mathrm{O}^{+}$ions and provide information on the distribution functions in the energy range from $\sim 25 \mathrm{eV}$ to $\sim 40 \mathrm{keV}$ every spin of the spacecraft (4 s). However, the calibrated data used are 
usually of lower time resolution (>4 s). All data are presented in the Geocentric Solar Magnetospheric (GSM) coordinate system except otherwise noted.

\subsection{Event observed on 5 August 2001}

Figure 1 shows a DF event observed by Cluster 1 (C1) on 5 August 2001. C1 was in the central plasma sheet ( $\beta>0.5$, not shown) at $X_{\mathrm{GSM}} \sim-17 R_{\mathrm{E}}$. In this figure, the $\mathrm{DF}$ is marked by the vertical black line at $\sim 14: 03: 39 \mathrm{UT}\left(t_{\mathrm{DF}}\right)$, when the increase of $B_{\mathrm{Z}}$ had the largest slope. $B_{\mathrm{z}}$ increased by $\sim 15 \mathrm{nT}$ in less than $10 \mathrm{~s}$ (Figure 1a), and there were no large-amplitude (> $5 \mathrm{nT}$ ) $B_{\mathrm{z}}$ disturbances in the $2 \mathrm{~min}$ time period before the DF was observed, indicating that the ambient plasma sheet was fairly quiet. A proton number density $\left(n_{\mathrm{H}+}\right)$ enhancement (Figure 1c) accompanied by the proton velocity $\left(V_{\mathrm{H}+}\right)$ increase (Figure 1f), was observed $\sim 1$ min prior to the DF arrival (vertical dashed black line). Behind the $\mathrm{DF}, n_{\mathrm{H}+}$ quickly dropped to a minimum and $T_{\mathrm{H}+}$ increased to a maximum in $\sim 8 \pm 4 \mathrm{~s}$ (vertical dashed blue line). Accordingly, flux depletion (flux dropout) was observed in the energy spectrum after the DF crossing (Figure 1h).

The O+ ions also exhibited variations associated with this DF. Before the DF arrival, $n_{\mathrm{O}+}($ Figure $1 \mathrm{~b})$ and $V_{\mathrm{O}+}\left(\right.$ Figure 1e) increased almost simultaneously with those of $\mathrm{H}^{+}$, but the percentage was larger with increase of $n_{\mathrm{O}+}$ about $\sim 100 \%$ (from $\sim 0.02 \mathrm{~cm}^{-3}$ to $\sim 0.04 \mathrm{~cm}^{-3}$, Figure $1 \mathrm{~b}$ ), while with $n_{\mathrm{H}+}$ it was only $\sim 17.5 \%$ (from $\sim 0.40 \mathrm{~cm}^{-3}$ to $\sim 0.47$ $\mathrm{cm}^{-3}$, Figure 1c). After the DF crossing, $n_{\mathrm{O}+}$ was reduced to a minimum value within $34 \pm 4 \mathrm{~s}$ (Figure 1b, vertical dashed red line), which was $\sim 4$ times longer than that of $n_{\mathrm{H}+}(\sim 8 \pm 4 \mathrm{~s})$. The $T_{\mathrm{O}+}$ reached a maximum when the $n_{\mathrm{O}+}$ decreased to its minimum (Figure 1d). Behind the DF, there was also a flux dropout in the $\mathrm{O}^{+}$energy spectrum (Figure 1g). The boundary (contour) is energy dependent but it was not as clear in $\mathrm{H}^{+}$ 
spectrum. Similar signatures were observed on C3 and C4 (not shown).

\subsection{Ion Flux Dropout}

We now present a detailed analysis of the observed behavior of $\mathrm{H}^{+}$and $\mathrm{O}^{+}$energy spectra and velocity distribution functions. First to note is that during the DF crossing, the four Cluster spacecraft formed a regular tetrahedron configuration with the elongation and planarity $\sim 0.084$ and $\sim 0.099$, respectively. We have estimated the propagating velocity of the DF by applying multi-spacecraft timing method [e.g., Schwartz, 1998], in which the DF is assumed to be a quasi-planar structure. This timing method yielded a speed of $\sim 190 \mathrm{~km} / \mathrm{s}\left(V_{\mathrm{DF}}\right)$ with propagating direction $\mathbf{k}$ along $\sim(0.91,0.37,-0.20)$. This speed is very similar to the plasma bulk velocities around the DF observed by each spacecraft, indicating that the bulk speed was stable during the four spacecraft crossing of the DF. This observation allows us to reconstruct the spatial structure of the DF by transforming the time-dependent data into space-dependent data. The results are shown in Figure 2, in which we define the horizontal-axis is $\delta x=\left(t-t_{\mathrm{DF}}\right) \times V_{\mathrm{DF}}$, to indicate the distance measured from the DF at $\delta x=0$ (marked by a black dotted line). Figures $2 \mathrm{~b}$ and $2 \mathrm{c}$ show the energy spectra for $\mathrm{O}^{+}$and $\mathrm{H}^{+}$, respectively. The $\mathrm{O}^{+}$spectrum shows a clear flux dropout behind the DF, which extends to larger distances at higher energies. At the upper energy limit of CODIF ( $40 \mathrm{keV})$, the $\mathrm{O}^{+}$flux dropout appeared in the fourth data point behind the $\mathrm{DF}(\sim 5000 \mathrm{~km})$, which is the place where $n_{\mathrm{O}+}$ reached minimum (red dotted line in Figure 1b). As noted earlier, the energy dependence of the $\mathrm{H}^{+}$flux dropout was not clear, with the flux reaching a minimum only in one data point (blue dotted line in Figure 1c), consistent with $n_{\mathrm{H}+}$ variations behind the DF.

In addition to the energy dependence of $\mathrm{O}^{+}$flux dropout, the dropout is also pitch 
angle dependent as shown in the pitch angle distributions of $\mathrm{O}^{+}$(Figures 2d, 2e, and 2f, each panel contains two energy channels of CODIF). The $\mathrm{O}^{+}$ions were generally parallel or anti-parallel to the magnetic field direction at the beginning after crossing the DF. Subsequently, in the same energy channels, some perpendicular components gradually appeared, indicating that particles with larger perpendicular velocities could reach further distance during the reflection process. All these features shown in Figure 2 are correlated with particle gyro-motion.

In the simulations of Zhou et al. [2010], the background plasma sheet ions are proposed to be accelerated and reflected in a half gyro-period after crossing the DF inside the dipolarization flux bundle (DFB). Hence, based on the information of magnetic field and pitch angle, we have estimated the gyro-radius of $\mathrm{O}^{+}$with different energies to represent the distances that the ions can reach behind the DF. The results are shown as red dots with error bars in Figure $2 \mathrm{~b}$. The width of the error bars in the $\mathrm{x}$-direction are determined from the energy channels and pitch angles, and the error bars in the vertical direction are the widths of energy channels. These red dots agree well with the contour of flux dropout, confirming the gyro-motion reflection process of $\mathrm{O}^{+}$. This feature is not clear for $\mathrm{H}^{+}$, because $\mathrm{H}^{+}$with an energy of $\sim 40 \mathrm{keV}$ (upper energy limit of CODIF) can only reach $1000 \mathrm{~km}$ behind the DF, and the time resolution of $\mathrm{H}^{+}$spectrum from CODIF was not adequate to observe this change.

Figure 3a schematically illustrates the equatorial trajectories of $\mathrm{O}^{+}$and $\mathrm{H}^{+}$during the reflection process in the DF rest frame. The vertical black line indicates the DF layer. In an ideal situation, particles in the ambient plasma sheet encounter the DF and perform a half gyro-motion inside the DFB. The gyro-motions of $\mathrm{O}^{+}$and $\mathrm{H}^{+}$(blue and red solid lines) result in the flux dropouts shown in Figure 2. The results in the $X$ 
displacement between their density minima shown in Figure 1 are due to the fact that the gyro-radius of $\mathrm{O}^{+}$is $\sim 4$ times larger than $\mathrm{H}^{+}$with the same energy. Figures $3 \mathrm{~b}$ and $3 \mathrm{c}$ represent the $\mathrm{O}^{+}$distributions in $V_{\text {para }}-V_{\text {perp1 }}$ and $V_{\text {perp1 }}-V_{\text {perp2 }}$ planes respectively recorded by C1 from 14:03:38 to 14:03:42 (near the DF). Similarly, Figures 3d and 3e show the $\mathrm{O}^{+}$distributions recorded by $\mathrm{C} 1$ from 14:03:46 to 14:03:50 (behind the DF). The direction of $V_{\text {perp2 }}$ is defined as the direction of $B \times V$, where $B$ is the magnetic field from FGM in GSE coordinate and $V$ is the velocity in GSE coordinate from the onboard moments. The $V_{\text {perp1 }}$ is defined as the direction of $V_{\text {perp } 2} \times B$. During this period, $V_{\text {perp } 1}$ is roughly earthward, and $V_{\text {perp2 }}$ is roughly duskward. These distributions (Figures $3 b$ to $3 e$ ) are transformed into the rest frame of DF. Figure $3 b$ and $3 d$ show that most ions enter into the DFB with higher parallel or anti-parallel components of velocity (as shown in Figure 2d-2f) but due to their gyro-motion in the DFB, the distributions include particles in the perpendicular directions. Figure $3 \mathrm{c}$ shows an ion population in the earthward direction $\left(+V_{\text {perp } 1}\right)$ with high velocity $(\sim 400 \mathrm{~km} / \mathrm{s})$. These ions will be those that have experienced acceleration and reflection in the DFB. Figure $3 \mathrm{e}$ shows a pronounced population in the earthward direction $\left(+V_{\text {perp1 }}\right)$ with a duskward component $\left(+V_{\text {perp } 2}\right)$, which agrees with the expected behavior of $\mathrm{O}^{+}$that reach the furthest distance behind the DF. However, the duskward deflected population was not observed in $\mathrm{H}^{+}$observations because the reflection process of $\mathrm{H}^{+}$ only occurs in less than one data point in CODIF observations (MMS observations [Burch et al., 2015], which have a much higher time resolution than Cluster might reveal this effect).

Figures $3 \mathrm{f}$ to $3 \mathrm{i}$ represent the pitch angle distributions around the DF crossing with a time resolution of $8 \mathrm{~s}$ (corresponding to the purple segments in Figure 2a). It is clearly shown that the energy limits of flux dropouts (marked by black arrows) increase 
gradually with time from Figures $3 \mathrm{f}$ to $3 \mathrm{i}$ and the total particle flux decreases successively especially in the parallel and anti-parallel directions. During this process, the particles are accelerated in the perpendicular direction by the DFB-associated duskward electric field [Zhou et al., 2010; Wu and Shay., 2012]. These figures also indicate that there is a much higher oxygen flux in the parallel and anti-parallel directions ahead of the DF. We propose that these $\mathrm{O}^{+}$ions are from the ionosphere. The upflowing $\mathrm{O}+$ are mainly moving parallel or anti-parallel to the background magnetic field. The highest $\mathrm{O}^{+}$particle flux is in the magnetic dip region (Figure 3f) compared with the others. This feature is consistent with the observations in Figure 1 that $n_{\mathrm{O}+}$ in the magnetic dip region was much higher than the densities in the ambient plasma sheet.

\subsection{Superposed epoch analysis}

To retrieve a common pattern of ion variations during the DFs crossing, we have performed a superposed epoch analysis of the DF observations [Ohtani et al., 2004; Slavin et al., 2003]. Using the same criteria as in the previous studies [Sun et al., 2013; Yao et al., 2013], DF crossings were selected based on the following critiral. (1) $\delta B_{\mathrm{z}}>$ $10 \mathrm{nT}$ in less than 30s. (2) $\left|B_{\mathrm{x}}\right|<10 \mathrm{nT}$ during the interval $|\delta t|<30 \mathrm{~s}$, and $\delta t=t-t_{\mathrm{DF}}$, where $t_{\mathrm{DF}}$ is the time when $B_{\mathrm{z}}$ increasing has the largest slope. (3) Large-amplitude (> $5 \mathrm{nT}) B_{\mathrm{z}}$ variations are absent within 2 min before DF crossing to ensure the background is relatively quiet. (4) The time resolutions of CODIF data are equal to or smaller than $8 \mathrm{~s}$. (5) DF should propagate in earthward direction. A total of 22 events were selected using the Cluster data from the years 2001 to 2004.

Figure 4 shows the variations of the magnetic field and ion parameters within \pm 120 s of $t_{0}$, where $t_{0}$ indicates the onset of positive $B_{\mathrm{z}}$ variation (marked by black dashed 
line). Here, we define the parameter $\delta N_{O}=N_{O}(t)-N_{O}\left(t_{b}\right)$ to reveal the variations more clearly, where $t_{\mathrm{b}}=-120 \mathrm{~s}$ is the start time and $N_{O}$ is the $\mathrm{O}^{+}$density. We can obtain the variations of other parameters in a similar way. Moreover, the variations of $\mathrm{O}^{+}$and $\mathrm{H}^{+}$density $\left(\delta N_{\mathrm{O}}\right.$ and $\left.\delta N_{\mathrm{H}}\right)$, and temperature $\left(\delta T_{\mathrm{O}}\right.$ and $\left.\delta T_{\mathrm{H}}\right)$ are normalized by the values at $t_{\mathrm{b}}$ (mean value of three points around the $t_{\mathrm{b}}$ is used to represent the background situation). The average values have been calculated based on 22 events with the magnetic field $\left(B_{\mathrm{z}}\right)$ of $1 \mathrm{~s}$ time resolution and plasma parameters $8 \mathrm{~s}$ time resolution, shown as black points. In each panel, there is one solid line connecting these points showing the average values, and the other two dotted lines indicating the standard deviations.

Figure 4a shows a typical asymmetric bipolar structure of $B_{\mathrm{z}}$ for a DF at $t_{0}$, including the sharp enhancement ( 12 nT) and the low-amplitude $B_{\mathrm{Z}} \operatorname{dip}(\sim-2 \mathrm{nT})$. Behind the front, $B_{\mathrm{z}}$ returns to the quiet value $B_{\mathrm{z}}\left(t_{\mathrm{b}}\right)$ gradually. The $\mathrm{O}^{+}$density first increases smoothly (up to a factor of 1.5 ) before the DF crossing, starting at $\mathrm{t} \approx-80 \mathrm{~s}$, and then drops to $\sim 0.5$ of the initial value at $\mathrm{t} \approx 32 \mathrm{~s}$ (Figure $4 \mathrm{~b}$ ). Similarly, the $\mathrm{H}^{+}$density increases by a factor of $\sim 1.2$, starting at $\mathrm{t} \approx-40 \mathrm{~s}$, and then decreases rapidly to a factor of $\sim 0.7$ at $\mathrm{t} \approx 8 \mathrm{~s}$ (Figure $4 \mathrm{c}$ ). The $\mathrm{O}^{+}$temperature increases gradually by a factor of $\sim 1.3$ (Figure $4 \mathrm{~d}$ ), and the $\mathrm{H}^{+}$temperature shows a steeper increase by a factor of $\sim 1.2$ (Figure 4e). Comparing with $\mathrm{H}^{+}$, the increase of $\mathrm{O}^{+}$density starts earlier and the average increased percentage is much higher. After the DF crossing, $\mathrm{O}^{+}$ions lasted $\sim 4$ times longer than $\mathrm{H}^{+}$and decreased to the lowest density level with the largest temperature value (red and blue dashed lines respectively). These statistical features of $\mathrm{O}^{+}$during the $\mathrm{DF}$ crossing are shown here for the first time. The signatures behind the DF are consistent with the event on 5 August 2001 (see Figure 1). The $V_{\mathrm{x}}$ enhancement of $\mathrm{O}^{+}$and $\mathrm{H}^{+}$behind the DF are also observed, with the mean values of 
$\sim 150 \mathrm{~km} / \mathrm{s}$ and $\sim 200 \mathrm{~km} / \mathrm{s}$, respectively (Figures $4 \mathrm{f}$ and $4 \mathrm{~g}$ ).

\section{Simulations}

To further investigate the process of $\mathrm{O}^{+}$acceleration and reflection at DFs, we have used the backward-tracing test-particle simulations [Zhou at al., 2011, 2014]. We start with the initial equilibrium ion distributions $f\left(r_{i}, v_{i}, t_{i}\right)$ of modeled self-consistent kinetic plasma sheet [e.g., Harris, 1962; Pritchett and Coroniti, 1995]. The distributions $f(r, v, t)$ at any time $t$ is determined by tracing the ion trajectories backward in time to obtain their locations $r_{i}$ and velocities $v_{i}$ at $t_{i}$ since the phase space density $f(r, v, t)$ is equal to $f\left(r_{i}, v_{i}, t_{i}\right)$ according to the Liouville's theorem [e.g., Schwartz et al.,1998; Wanliss et al., 2002].

We applied the same initial equilibrium model previously used in Zhou et al. [2011, 2014] and calculated the reflection process of $\mathrm{H}^{+}$and $\mathrm{O}^{+}$assuming the density and thermal energy are similar as in the initial distributions. Other parameters included $L$, the plasma sheet half-thickness, equal $0.5 \mathrm{R}_{\mathrm{E}} ; B_{n}$, the neutral sheet $B_{z}$, equals to $2 \mathrm{nT}$. Although the model can be refined to accommodate a higher $B_{z}$ closer to the earth similar to the distribution of Earth's dipole field [Zhou et al., 2012], here we only use a simple model without a dipole to isolate the reflection process from the complications of the equatorial $B_{z}$ gradient effect. The $B_{0}$, the lobe magnetic field strength at $x_{0}$, equal to $30 \mathrm{nT}, n_{0}$, the equatorial plasma density at $x_{0}$, equal to 0.35 $\mathrm{cm}^{-3}$ (for both simulations of $\mathrm{H}^{+}$and $\mathrm{O}^{+}$), and $v_{T}$, the ion thermal velocity within the entire plasma sheet, equal to $700 \mathrm{~km} / \mathrm{s}$ and $250 \mathrm{~km} / \mathrm{s}$ for $\mathrm{H}^{+}$and $\mathrm{O}^{+}$respectively. Furthermore, a cold $\left(v_{T}=400 \mathrm{~km} / \mathrm{s}\right.$ for $\mathrm{H}^{+}$and $v_{T}=100 \mathrm{~km} / \mathrm{s}$ for $\left.\mathrm{O}^{+}\right)$, tenuous $\left(n_{0}=\right.$ $0.05 \mathrm{~cm}^{-3}$ ) and non-drifting population was superposed over the entire plasma sheet to represent the background cold plasma and the lobe limit. Finally, we assumed that 
there were no initial distributions behind the DF by setting $f\left(r_{i}, v_{i}, t_{i}\right)=0$ where $x_{i}$ was less than the initial $x$ location of DF. It should be mentioned that ions originating from the distant tail or lobe may also contribute to the accelerated populations in the DFB and even to the precursor populations ahead of the DF, which have been revealed in both PIC [Eastwood et al., 2015] and test particle [Birn et al., 2017] simulations. However, these populations will not influence the reflection process of particles near the front. Therefore, we have excluded these populations in the present study.

An earthward propagating DF-associated electromagnetic field was superimposed over the initial equilibrium background and the DF is assumed to be a plane with step-like enhancements of the magnetic and electric fields. Then the magnetic perturbation is given by

$$
\Delta B_{z}(x, y, t)=\frac{B_{f}}{2}\left[1-\tanh \left(\frac{x^{*}}{L_{f}}\right)\right] \exp \left(-\frac{y^{2}}{{H_{f}}^{2}}\right),
$$

where $x^{*}=x-x_{f}-v_{f}\left(t-t_{i}\right)$ describes the earthward propagation of DF at the speed of $v_{f}$, which equals $200 \mathrm{~km} / \mathrm{s}$ in our simulations. Here, $B_{f}$ is the maximum $B_{z}$ enhancement across the DF; $x_{f}$ is the initial $\mathrm{x}$ location of DF at $t=t_{i} ; L_{f}$ is the DF half-thickness; and $H_{f}$ is the half-width of DF in y direction. For comparison with our case study, the adopted parameters used are as follows: $B_{f}=18 \mathrm{nT}, t_{i}=0 \mathrm{~s}, L_{f}=0.1 R_{E}$, and $H_{f}=2 R_{E}$. There is also a dawn-dusk electric field carried by the earthward propagating DF to satisfy the Faraday's law

$$
E_{y}(x, y, t)=v_{f} \Delta B_{z}(x, y, t)
$$

that can be considered as a superposition of an induced static potential electric field (see more details in Zhou et al. [2014]).

Based on the model specified above, we then calculated the ion distributions at any 
arbitrary location by tracing the ion trajectories backward in time. For this calculation, we placed a virtual spacecraft in the central plasma sheet at $(-10,0,0) R_{E}$, then simulated energy spectra of $\mathrm{O}^{+}$and $\mathrm{H}^{+}$at this location shown in Figure 5a and 5c. The virtual spacecraft encounters the earthward propagating DF at $t \sim 32 \mathrm{~s}$. For purposes of comparison, energy spectra of $\mathrm{O}^{+}$and $\mathrm{H}^{+}$on 5 August 2001 are also shown in Figures $5 \mathrm{~b}$ and $5 \mathrm{~d}$. As before, the horizontal axis has been plotted as spatial scale (section 2.2). Therefore, the horizontal-axis $\delta x$ indicate the distances from the DF, and the DF crossing has been marked by a white vertical line at $\delta x=0$.

The flux dropouts are clearly observed in our simulated energy flux spectra (Figures $5 \mathrm{a}$ and $5 \mathrm{c}$ ), which show contours similar to observations (Figures $5 \mathrm{~b}$ and $5 \mathrm{~d}$ ). We superposed the solid curves in each panel to show the gyro-radii of ions with different energies (as mentioned in Figure 2b). The solid curve is approximately consistent with the flux dropout in each panel. To investigate the reflection process, we have compared the orbits of those particles that contributed the most to the energy flux around the curves. Figure $6 \mathrm{a}-6 \mathrm{~d}$ show the typical orbits of $\mathrm{O}^{+}$with different energies that contributed to the duskward beam around the furthest locations away from the DF (corresponding to the black point marked by $a-d$ respectively in Figure 5a). The points $a-c$ are selected along the solid curve and the particle orbits at these locations are plotted in Figure 6a-6c. Obviously we can find out that particles indeed experienced a half gyro-motion after encountering the DF, with the encountering moments marked by the red stars, the moving directions marked by the red arrows, and their gyroradii increase as they gain energy during the process.

Note that although the main populations cross the DF along the normal direction and perform a half gyro-motion in DFB because of the high-speed earthward propagation 
of DF, there are also some ions (e.g., ions marked by $d$ in Figure 5a) that spend more than a half period of gyration in the DFB depending on their specific incident angle. The corresponding typical orbits are shown in Figure 6d. Accordingly, Figure 6e schematically illustrates an equatorial trajectory of the $\mathrm{O}^{+}$that encounters the DF with a smaller incident angle (marked by the dotted curve). These ions can reach a further distance during the reflection process than those ions only performing a half gyro-motion (marked by the solid curve in Figure 6e). Therefore, we recalculated the largest depth of the ions with different energy during their gyro-motion behind the DF corresponding to this situation (i.e., the incident angle is $45^{\circ}$, the typical orbit is corresponding to the dotted trajectory illustrated in Figure 6e). The results are marked by dotted curves in Figure 5a-5d. In the $\mathrm{O}^{+}$spectra of both observation and simulation, the contours of dropouts indeed arrived to deeper locations near the dotted curves in the high-energy range. It also indicates $\mathrm{O}^{+}$ions with higher velocities are easier to deflect from the normal direction (incident angle $\sim 90^{\circ}$ ) when they encounter the DF. For $\mathrm{H}^{+}$, some ions gyrate beyond the curves and can arrive at deeper locations (Figure $5 \mathrm{c})$, which are contributed by the species that had a smaller incident angle $\left(<45^{\circ}\right)$.

\section{Discussion}

Our observations and simulations have provided a detailed understanding of the reflection process associated with DFs. The DFB propagates earthward and encounters the ambient plasma sheet ions. These ions penetrate into the DFB, and then accelerated and reflected by the DFB. During the reflection process, the injected ions with different initial energies and pitch angles will reach different positions behind the DF. For the same ion species with the same specific charge, particles with higher energy and closer to $90^{\circ}$ pitch angle can penetrate to further distances from the DF. Hence, the spacecraft would detect fewer particles as it travels to deeper locations 
inside the DFB, leading to a gradual decrease in the ion density. This reflection process is clearly visible in $\mathrm{O}^{+}$properties in our case study, but not in $\mathrm{H}^{+}$. Taking into consideration that $\mathrm{O}^{+}$and $\mathrm{H}^{+}$share the same energy upper limit of CODIF, the gyro-radius of $\mathrm{O}^{+}$with the highest energy would be 4 times larger than that of $\mathrm{H}^{+}$, producing the observational signature of the $\mathrm{O}^{+}$density minimum that appears $\sim 4$ times later than that of $\mathrm{H}^{+}$after the DF crossing.

We have shown statistically ion property variations during DF crossings (Figure 4). The $\mathrm{H}^{+}$signatures in Figure 4 are similar to the results (also statistical) in Runov et al. [2011] (see their Figure 5). Similar to our results, in Runov et al. [2011], the ion density increased gradually ahead of the front, starting at $\mathrm{t} \sim-40 \mathrm{~s}$, and then dropped rapidly to a factor of $\sim 0.5$. However, the ion temperature increased gradually to a factor of 1.6 behind the front, which is higher than that of the $\mathrm{H}^{+}$in our events. The difference may be because the ion moments used in Runov et al. [2011] are calculated for a much broader energy range $(\sim 5 \mathrm{eV}$ to $1 \mathrm{MeV})$, while the proton moments used in this study only included particles with energy lower than $40 \mathrm{keV}$. From Figure 4, we also find that the precursor signatures of $\mathrm{O}^{+}$are more prominent and appear earlier than that of $\mathrm{H}^{+}$. Precursor signatures are explained in the picture of ion acceleration and reflection at DFs [e.g., Zhou et al., 2010, 2011]. Accordingly, these differences may also be caused by the larger gyro-radii of $\mathrm{O}^{+}$. However, these are not the main points in this paper, so more details will be revealed in the future work.

\section{Conclusion}

This paper has analyzed the Cluster data and together with test particle simulations studied the variations of $\mathrm{O}^{+}$behavior around the DF in the Earth's plasma sheet. The 
main points can be summarized as follows:

1. After the crossing of a DF, the $\mathrm{O}^{+}$density decreases more gradually than $\mathrm{H}^{+}$ density. And the distance between the density minimum of $\mathrm{O}^{+}$and the DF layer is $\sim 4$ times longer than that of $\mathrm{H}^{+}$.

2. The density minimum position in the DFB can be understood as the farthest position where the particles performing gyro-motions can reach. These particles have the highest energy and their pitch angle are close to $90^{\circ}$. Hence, $\mathrm{O}^{+}$can extend to a deeper position in the DFB due to its larger gyro-radius than that of $\mathrm{H}^{+}$ with the same energy.

3. The flux dropout in $\mathrm{O}^{+}$energy spectrum is shown in both observations and simulations. The contour of flux dropout is energy-dependent and highly correlated with the gyro-radii of $\mathrm{O}^{+}$ions with different energies, which further supports that $\mathrm{O}^{+}$is reflected in a half gyro-motion in the DFB.

\section{Acknowledgements.}

We are grateful to Cluster FGM, CIS teams, and CSA Archive (http://www.cosmos.esa.int/web/csa/) for providing the data. This research has been supported by the National Natural Science Foundation of China (grants 41474139, 41731068, and 41704163).

\section{Reference}

Balogh, A., et al. (2001), The Cluster magnetic field investigation: Overview of in-flight performance and initial results, Ann. Geophys., 19,1207-1217.

Birn, J., A. Runov, and X.-Z. Zhou (2017), Ion velocity distributions in dipolarization events: Distributions in the central plasma sheet, J. Geophys. Res. Space Physics, 122, 8014-8025, 
doi:10.1002/2017JA024230.

Burch, J. L., T. E. Moore, R. B. Torbert, and B. L. Giles (2015), Magnetospheric multiscale overview a nd science objectives, Space Sci. Rev., 1-17, doi:10.1007/s11214-015-0164-9.

Eastwood, J. P., M. V. Goldman, H. Hietala, D. L. Newman, R. Mistry, and G. Lapenta (2015), Ion reflection and acceleration near magnetotail dipolarization fronts associated with magnetic reconnection, J. Geophys. Res. Space Physics, 120, 511-525, doi:10.1002/2014JA020516.

Escoubet, C. P., M. Fehringer, and M. Goldstein (2001), Introduction: The Cluster mission, Ann. Geophys., 19, 1197-1200.

Fu, H. S., et al. (2012), Electric structure of dipolarization front at sub-proton scale, Geophys. Res. Lett., 39(L06105).

Fu, S. Y., B. Wilken, Q.-G. Zong, and Z. Y. Pu (2001), Ion composition variation in the inner magnetosphere-individual and collective storm effects in 1991, J. Geophys. Res., 106, 29,683-29,698.

Fu, S. Y., et al. (2011), High-speed flowing plasmas in the Earth's plasma sheet, Chinese Sci. Bull., 56(12), 1182-1187.

Greco, A., A. Artemyev, and G. Zimbardo (2015), Heavy ion acceleration at dipolarization fronts in planetarymagnetotails, Geophys. Res. Lett., 42, 8280-8287, doi:10.1002/2015GL066167.

Harris, E. G. (1962), On a plasma sheet separating regions of oppositely directed magnetic field, Nuovo Cimento, 23, 115-121.

Kistler, L. M., et al. (2005), Contribution of nonadiabatic ions to the cross-tail current in an O+ dominated thin current sheet, J. Geophys. Res., 110, A06213, doi:10.1029/2004JA010653.

Liang, H., G. Lapenta, R. J. Walker, D. Schriver, M. El-Alaoui, and J. Berchem (2017), Oxygen acceleration in magnetotail reconnection, J. Geophys. Res. Space Physics, 122, doi:10.1002/2016JA023060.

Liu, J., V. Angelopoulos, A. Runov, and X.-Z. Zhou (2013), On the current sheets surrounding dipolarizing flux bundles in the magnetotail: The case for wedgelets, J. Geophys. Res. Space Physics, 118, 2000-2020, doi:10.1002/jgra.50092. 
Liu, J., V. Angelopoulos, X. Chu, X.-Z. Zhou, and C. Yue (2015), Substorm current wedge composition by wedgelets, Geophys. Res. Lett., 42, 1669-1676, doi:10.1002/2015GL063289.

Lu, S., A. V. Artemyev, V. Angelopoulos, Q. Lu, and J. Liu (2016), On the current density reduction ahead of dipolarization fronts, J. Geophys. Res. Space Physics, 121, 4269-4278, doi:10.1002/2016JA022754.

Nakamura, R., et al. (2002), Motion of the dipolarization front during a flow burst event observed by Cluster, Geophys. Res. Lett., 29(20), 1942, doi:10.1029/2002GL015763.

Nilsson, H., M. Hamrin, T. Pitkänen, T. Karlsson, R. Slapak, L. Andersson, H. Gunell, A. Schillings, and A. Vaivads (2016), Oxygen ion response to proton bursty bulk flows, J. Geophys. Res. Space Physics, 121, 7535-7546, doi:10.1002/2016JA022498.

Ohtani, S. I., M. A. Shay, and T. Mukai (2004), Temporal structure of the fast convective flow in the plasma sheet: Comparison between observations and two-fluid simulations, J. Geophys. Res., 109, A03210, doi:10.1029/2003JA010002.

Pan, D.-X., X.-Z. Zhou, Q.-Q. Shi, J. Liu, V. Angelopoulos, A. Runov, Q.-G. Zong, and S.-Y. Fu (2015), On the generation of magnetic dips ahead of advancing dipolarization fronts, Geophys. Res. Lett., 42, 4256-4262, doi:10.1002/2015GL064369.

Pritchett, P. L., and F. V. Coroniti (1995), Formation of thin current sheets during plasma sheet convection, J. Geophys. Res., 100,

$23,551-23,565$.

Rème, H., et al. (2001), First multispacecraft ion measurements in and near the Earth's magnetosphere with the identical Cluster ion spectrometry (CIS) experiment, Ann. Geophys., 19, 1303-1354.

Runov, A., V. Angelopoulos, M. I. Sitnov, V. A. Sergeev, J. Bonnell, J. P. McFadden, D. Larson, K.-H. Glassmeier, and U. Auster (2009), THEMIS observations of an earthward-propagating dipolarization front, Geophys. Res. Lett., 36, L14106, doi:10.1029/2009GL038980.

Runov, A., V. Angelopoulos, X.-Z. Zhou, X.-J. Zhang, S. Li, F. Plaschke, and J. Bonnell (2011), A THEMIS multicase study of dipolarization fronts in the magnetotail plasma sheet, J. Geophys. Res., 116, A05216, doi:10.1029/2010JA016316.

Schwartz, S. J. (1998), Shock and discontinuity normals, Mach numbers and related parameters, in 
Analysis Methods for Multi-Spacecraft Data, edited by G. Paschmann and and P. W. Daly, pp. 249270, Int. Space Sci. Inst., Bern, German.

Seki, K., R. C. Elphic, M. Hirahara, T. Terasawa, and T. Mukai (2001), On atmospheric loss of oxygen ions from Earth through magnetospheric processes, Science, 291, 1939-1941.

Sergeev, V., V. Angelopoulos, S. Apatenkov, J. Bonnell, R. Ergun, R. Nakamura, J. McFadden, D. Larson, and A. Runov (2009), Kinetic structure of the sharp injection/dipolarization front in the flow braking region, Geophys. Res. Lett., 36, L21105, doi:10.1029/2009GL040658.

Shay, M. A., and M. Swisdak (2004), Three-species collisionless reconnection: Effect of O+ on magnetotail reconnection, Phys. Rev. Lett., 93(17), 175001, doi:10.1103/PhysRevLett.93.175001.

Slavin, J. A., et al. (2003), Geotail observations of magnetic flux ropes in the plasma sheet, J. Geophys. Res., 108(A1), 1015, doi:10.1029/2002JA009557.

Sun, W.-J., S. Y. Fu, G. K. Parks, J. Liu, Z. H. Yao, Q. Q. Shi, Q.-G. Zong, S. Y. Huang, Z. Y. Pu, and T. Xiao (2013), Field-aligned currents associated with dipolarization fronts, Geophys. Res. Lett., 40, 4503-4508, doi:10.1002/grl.50902.

Sun, W.-J., S. Fu, G. K. Parks, Z. Pu, Q.-G. Zong, J. Liu, Z. Yao, H. Fu, and Q. Shi (2014a), Electric fields associated with dipolarization fronts, J. Geophys. Res. Space Physics, 119, 5272 - 5278, doi:10.1002/2014JA020045.

Sun, W. J., S. Fu, Z. Pu, G. K. Parks, J. A. Slavin, Z. Yao, Q.-G. Zong, Q. Shi, D. Zhao, and Y. Cui (2014b), The current system associated with the boundary of plasma bubbles, Geophys. Res. Lett., 41, 8169 - 8175, doi:10.1002/2014GL062171.

Wanliss, J. A., R. D. Sydora, G. Rostoker, and R. Rankin (2002), Origin of some anisotropic tailward flows in the plasma sheet, Ann. Geophys., 20, 1559-1575.

Wu, P., and M. A. Shay (2012), Magnetotail dipolarization front and associated ion reflection: Particle-in-cell simulations, Geophys. Res. Lett., 39, L08107, doi:10.1029/2012GL051486.

$\mathrm{Wu}, \mathrm{T}$., et al. (2016), Thin energetic O+ layer embedded in the magnetotail reconnection current sheet observed by Cluster, Geophys. Res. Lett., 43, 11,493-11,500, doi:10.1002/2016GL071184.

Yao, Z. H., et al. (2013), Current structures associated with dipolarization fronts, J. Geophys. Res. Space Physics, 118, 6980-6985, doi:10.1002/2013JA019290. 
Yao, Z. H., et al. (2015), A physical explanation for the magnetic decrease ahead of depolarization fronts, Ann. Geophys., 33, 1301-1309.

Zelenyi, L. M., et al. (2006), "Matreshka" model of multilayered current sheet, Geophys. Res. Lett., 33, L05105, doi:10.1029/2005GL025117.

Zhao, D., et al. (2016), Electromagnetic disturbances observed near the dip region ahead of dipolarization front, Geophys. Res. Lett., 43, 3026-3034 doi:10.1002/2016GL068033.

Zhou, X.-Z., V. Angelopoulos, V. A. Sergeev, and A. Runov (2010), Accelerated ions ahead of earthward-propagating dipolarization fronts, J. Geophys. Res., 115, A00I03, doi:10.1029/2010JA015481.

Zhou, X.-Z., V. Angelopoulos, V. A. Sergeev, and A. Runov (2011), On the nature of precursor flows upstream of advancing of dipolarization fronts, J. Geophys. Res., 116, A03222, doi:10.1029/2010JA016165.

Zhou, X.-Z., Angelopoulos, V., Runov, A., Liu, J., \& Ge, Y. S. (2012). Emergence of the active magnetotail plasma sheet boundary from transient, localized ion acceleration. Journal of Geophysical Research, 117, A10216. https://doi.org/10.1029/2012JA018171

Zhou, X.-Z., V. Angelopoulos, J. Liu, A. Runov, and S.-S. Li (2014), On the origin of pressure and magnetic perturbations ahead of dipolarization fronts, J. Geophys. Res. Space Physics, 119, 211 - 220, doi:10.1002/2013JA019394.

\section{Figure captions}

Figure 1. Overview of the magnetic field and ion observations from Cluster-C1 for the dipolarization front (DF) on 5 August 2001. (a) magnetic field components, $B_{\mathrm{x}}$ (blue), $B_{\mathrm{y}}$ (green), $B_{\mathrm{z}}$ (red); (b) $\mathrm{O}^{+}$density, $n_{\mathrm{O}+}$; (c) $\mathrm{H}^{+}$density, $n_{\mathrm{H}+}$; (d) Ion temperature, $T_{\mathrm{O}+}(\mathrm{red}), T_{\mathrm{H}+}$ (blue); (e) $\mathrm{O}^{+}$velocity components, $V_{\mathrm{x}}$ (blue), $V_{\mathrm{y}}$ (green), $V_{\mathrm{z}}$ (red); (f) $\mathrm{H}^{+}$velocity components, $V_{\mathrm{x}}$ (blue), $V_{\mathrm{y}}$ (green), $V_{\mathrm{z}}\left(\right.$ red); (g) $\mathrm{O}^{+}$and $(\mathrm{h}) \mathrm{H}^{+}$ energy spectrum for differential particle flux. The black dashed vertical line marks the time when $\mathrm{O}^{+}$and $\mathrm{H}^{+}$observed density enhancements prior to the DF. The black 
vertical line indicates the DF where $B_{\mathrm{z}}$ has the largest slope, and the blue and red dashed vertical lines indicate the minima of $n_{\mathrm{H}+}$ and $n_{\mathrm{O}+}$ behind the DF, respectively. Note that the time resolution for ion moments from CODIF for this observation was 8 s.

Figure 2. Observations and analysis of the $\mathrm{O}^{+}$and $\mathrm{H}^{+}$flux dropout. (a) magnetic field components; (b) $\mathrm{O}^{+}$and (c) $\mathrm{H}^{+}$energy spectra of the differential particle flux; $\mathrm{O}^{+}$pitch angle distributions in the energy range (d) from $\sim 23.7 \mathrm{keV}$ to $\sim 38.3 \mathrm{keV}$, (e) from $14.6 \mathrm{keV}$ to $\sim 23.7 \mathrm{keV}$, and (f) from $\sim 9.0 \mathrm{keV}$ to $\sim 14.6 \mathrm{keV}$. The black vertical dashed line marks $\delta x=0$, i.e., the time of largest $B_{\mathrm{Z}}$ slope. The red dotted line in (b) represent the distances that $\mathrm{O}^{+}$can reach. The error bars in the $\mathrm{x}$-direction are determined from the widths of the corresponding energy channels and pitch angles, and the error bars in the vertical direction are the widths of energy channels.

Figure 3. On the $\mathrm{O}^{+}$and $\mathrm{H}^{+}$reflections in the DF. (a) Schematic illustration of the $\mathrm{O}^{+}$ (red) and $\mathrm{H}^{+}$(blue) trajectories during the DF encounters in the DF rest frame; $V_{\text {para }}-V_{\text {perpl } 1}$ and $V_{\text {perp } 1}-V_{\text {perp } 2}$ cuts of the 3-D O ${ }^{+}$distributions at the $\mathrm{DF}(\mathrm{b}, \mathrm{c})$ and near the density minimum behind the DF (d, e); (f, g, h, i) $\mathrm{O}^{+}$particle flux distributions as a function of energy and pitch angle in four time ranges, corresponding to the purple segments in Figure 2a. The black arrows in $(g, h, i)$ show the energy limit of flux dropouts increasing gradually.

Figure 4. Superposed epoch analysis of the magnetic field (1s sampling rate) and particle parameters (8s sampling rate). Mean value (thick solid lines) with standard deviation (dotted lines) of (a) magnetic field; (b) $\mathrm{O}^{+}$and (c) $\mathrm{H}^{+}$normalized density variations; (d) $\mathrm{O}^{+}$and (e) $\mathrm{H}^{+}$normalized temperature variations; and $\mathrm{x}$ component of 
velocity for (f) $\mathrm{O}^{+}$and $(\mathrm{g}) \mathrm{H}^{+}$. The front crossing time is marked by the black vertical dotted line. The low-density with temperature maximum of $\mathrm{O}+$ and $\mathrm{H}+$ are marked by the red and blue dotted lines respectively.

Figure 5. Comparison between simulations and observations on the dropout. (a) Simulated and (b) observational for $\mathrm{O}^{+}$energy flux; (c) Simulated and (d) observational for $\mathrm{H}^{+}$energy flux. In (a, b, c, d), the vertical solid line marks $\delta x=0$, the solid curves correspond to the distances that different energies $\mathrm{O}^{+}$and $\mathrm{H}^{+}$can reach with incidence the angle of $90^{\circ}$, the dotted curves correspond to the distances that different energies $\mathrm{O}^{+}$and $\mathrm{H}^{+}$can reach with an incidence angle of $45^{\circ}$.

Figure 6. Typical orbits of $\mathrm{O}^{+}$around the dropout contour. (a - d) Typical orbits of $\mathrm{O}^{+}$ at the location corresponding to the black point marked by a-d respectively in Figure 5a; (e) Schematical illustration of the ion trajectories with an incidence angle of $90^{\circ}$ (solid curve) and $45^{\circ}$ (dotted curve) respectively during the DF encounters in the DF rest frame. In (a, b, c, d), The red star marks the DF encounters. The red arrow indicates the direction of $\mathrm{O}^{+}$motion. 


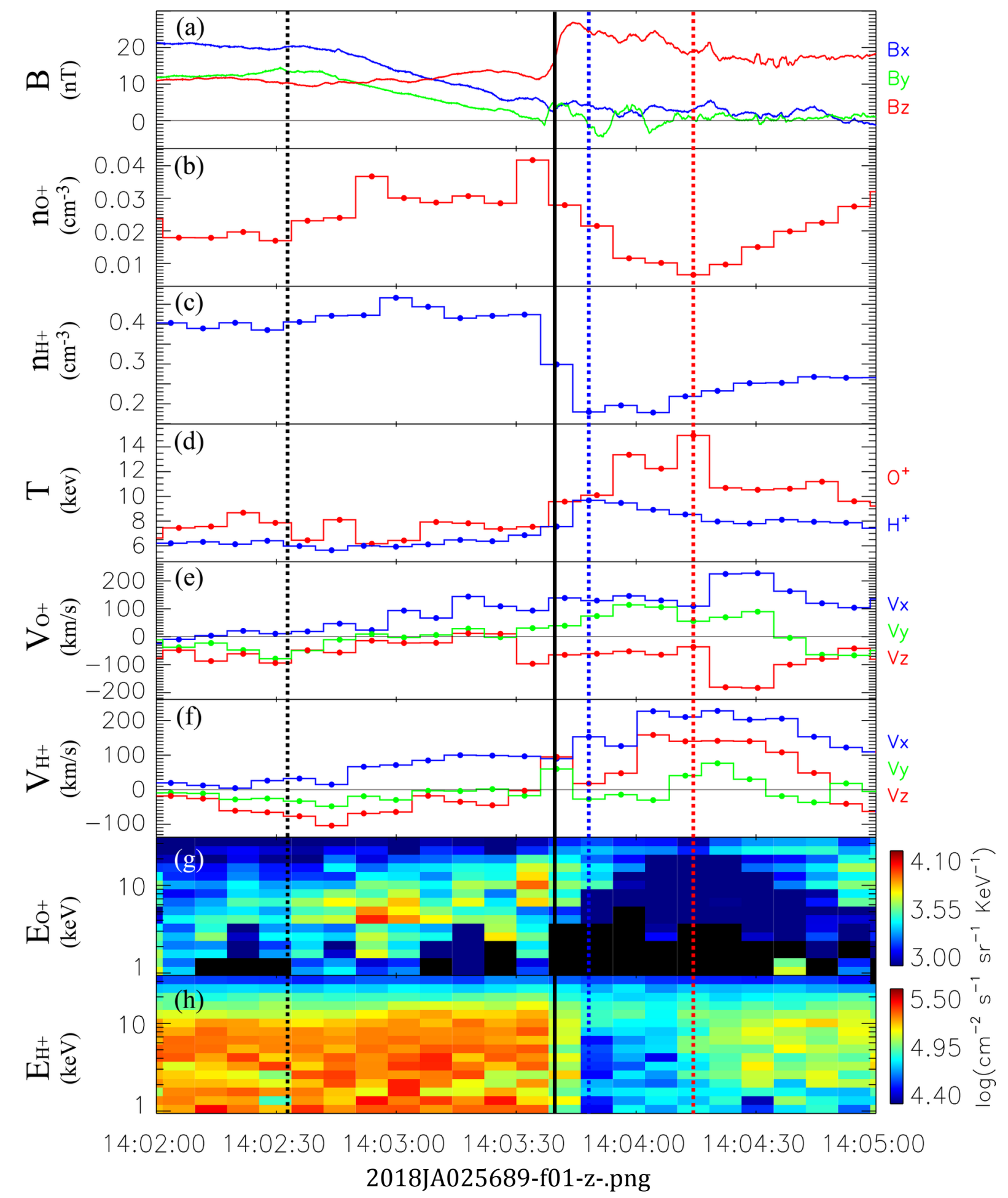

This article is protected by copyright. All rights reserved. 


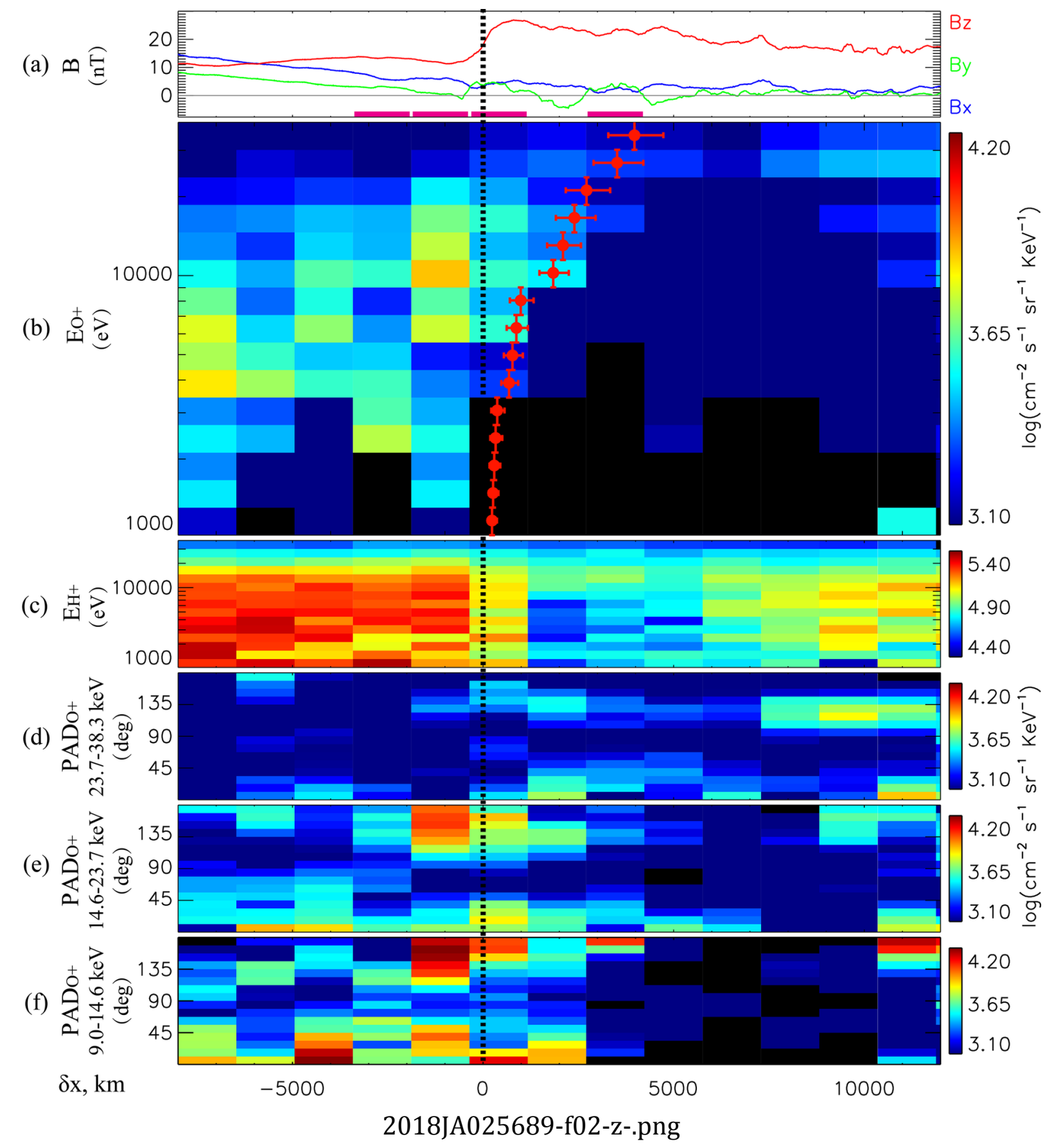

This article is protected by copyright. All rights reserved. 

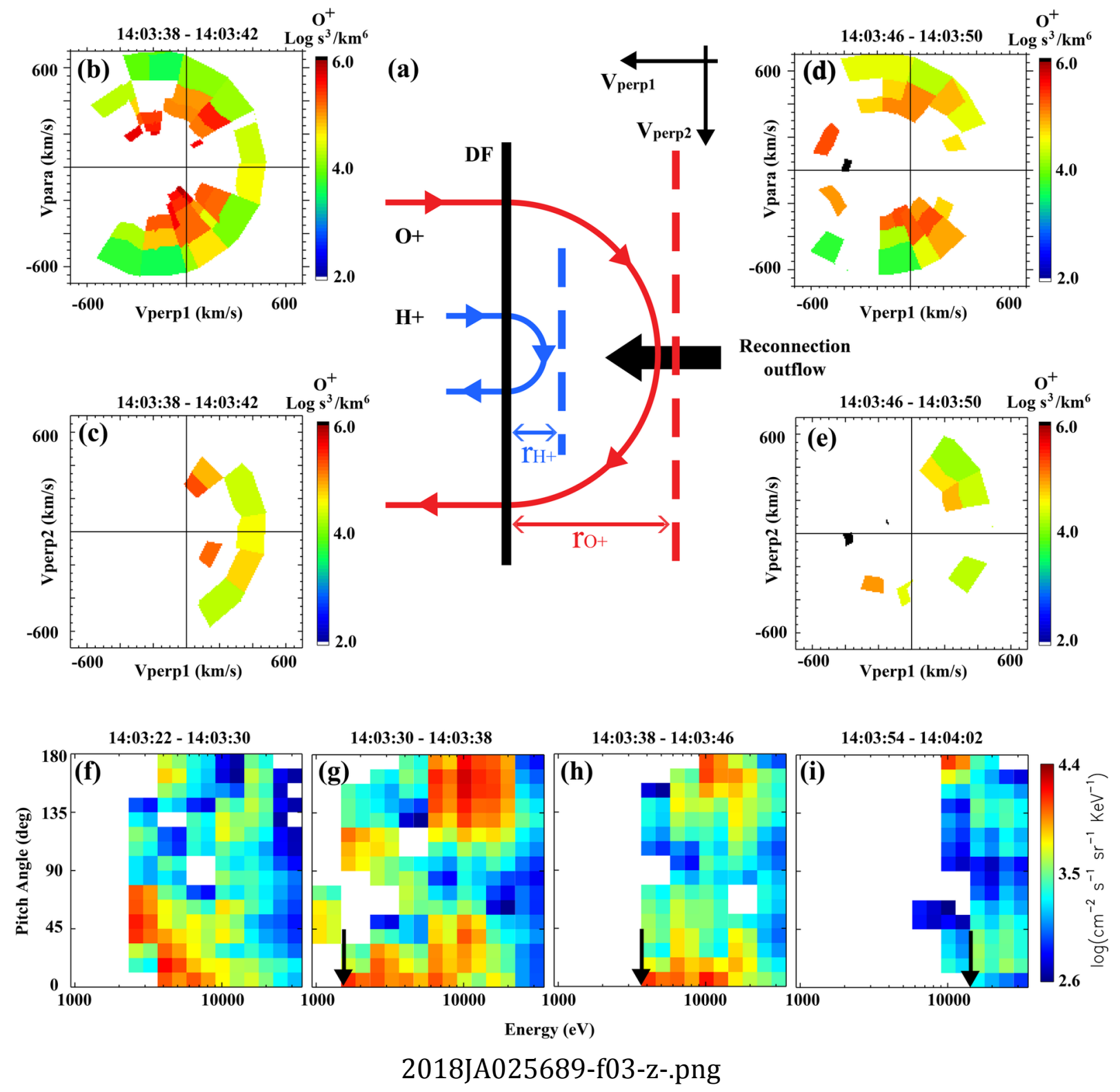

This article is protected by copyright. All rights reserved. 


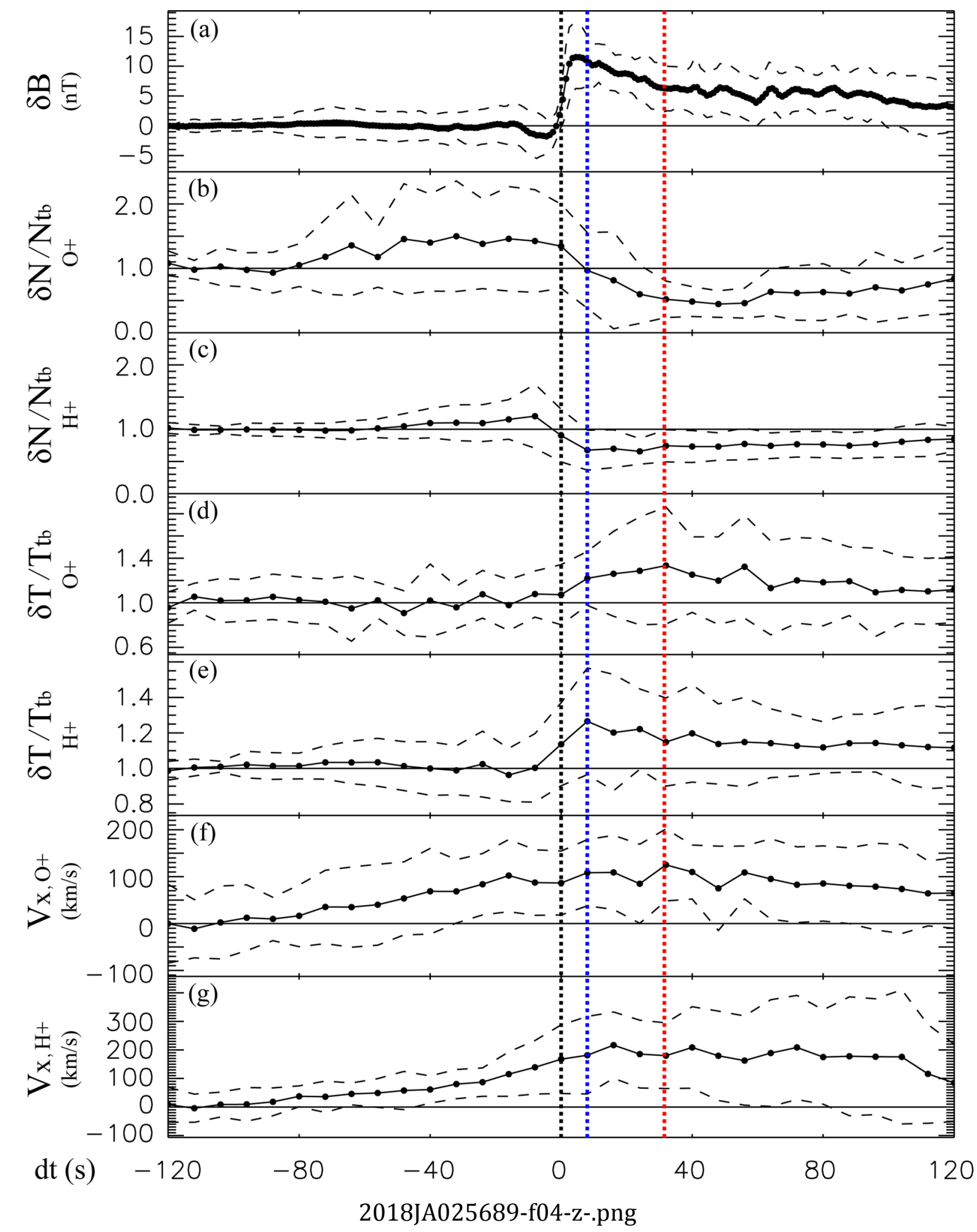

This article is protected by copyright. All rights reserved. 


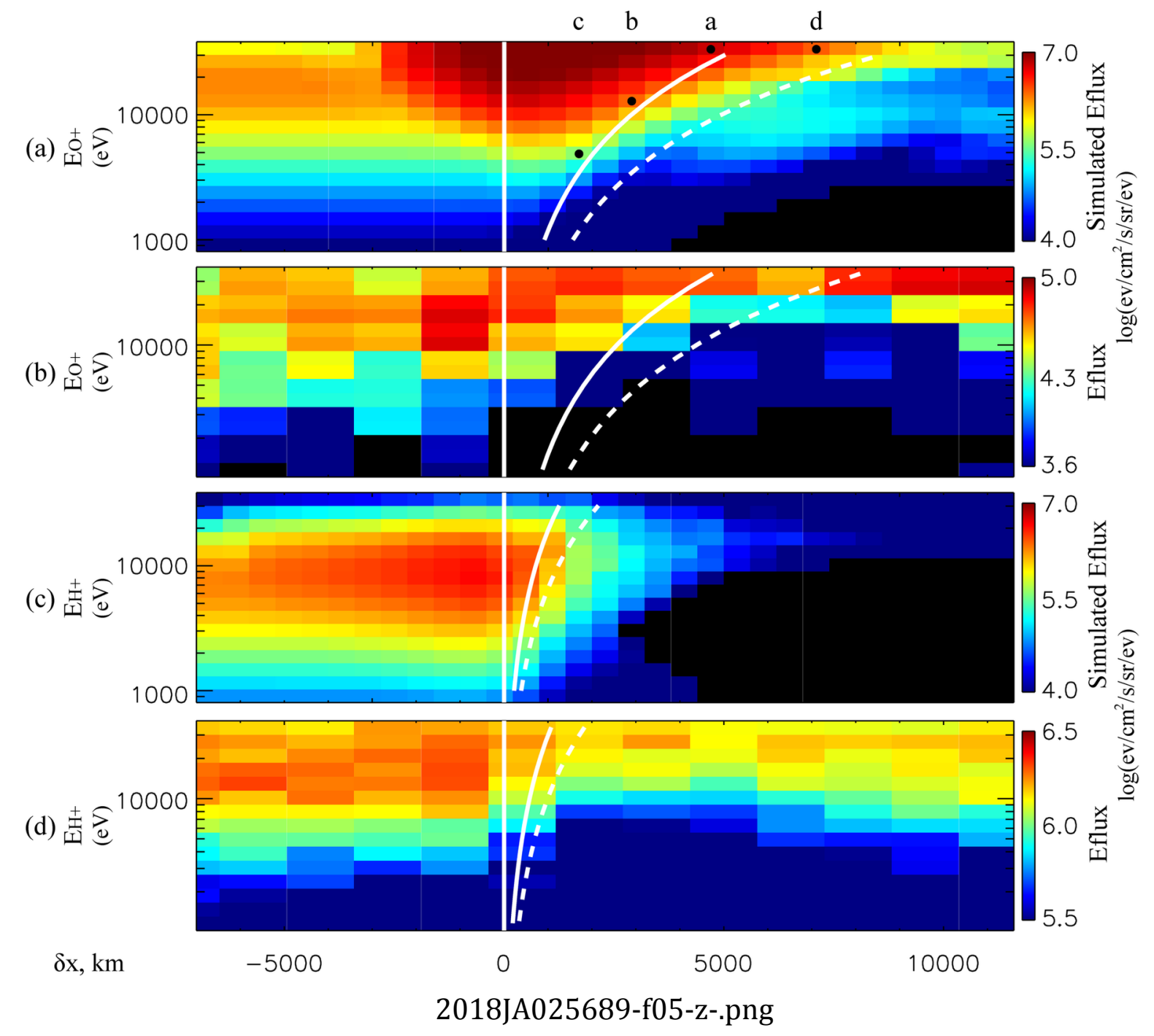

This article is protected by copyright. All rights reserved. 

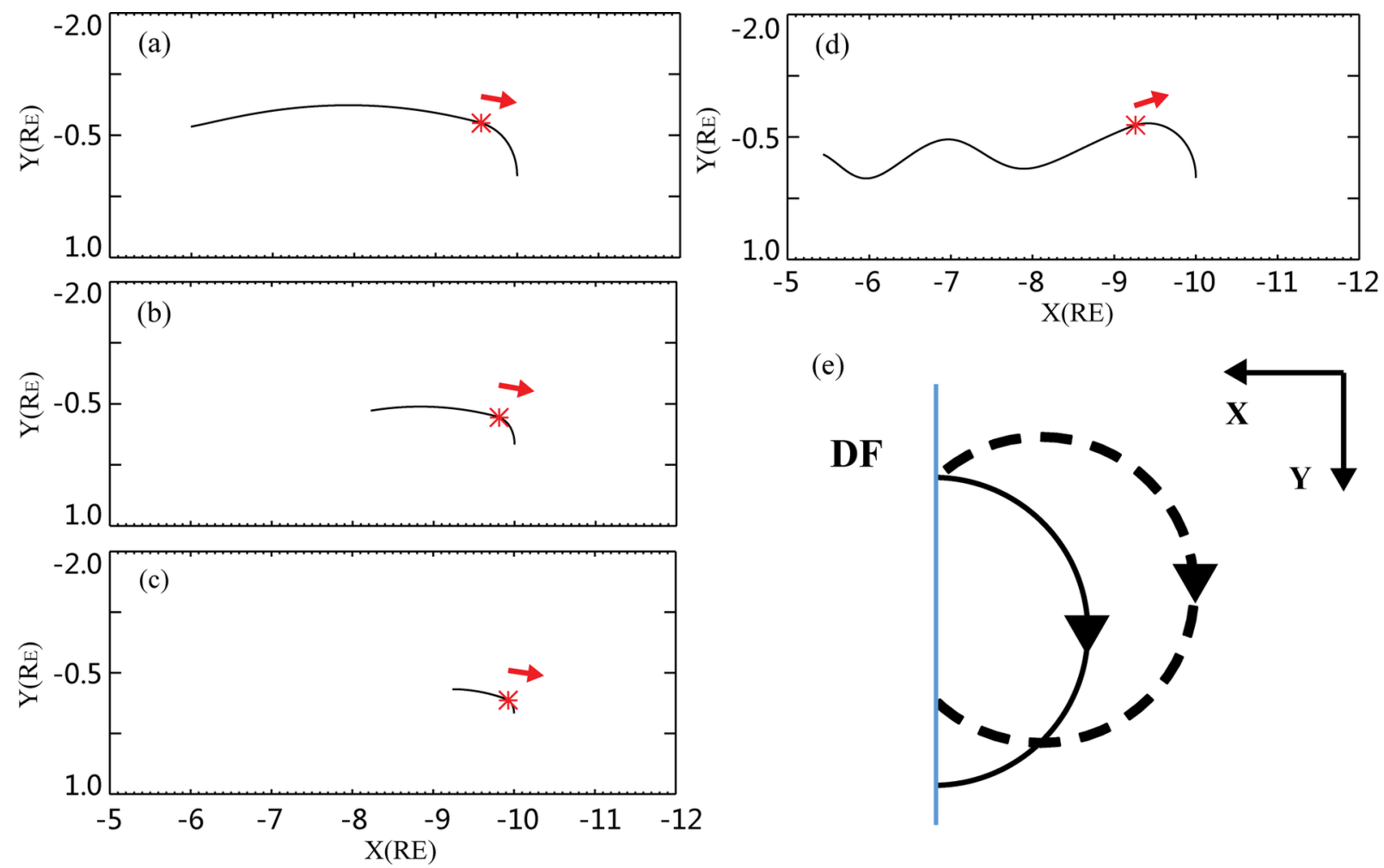

(e)

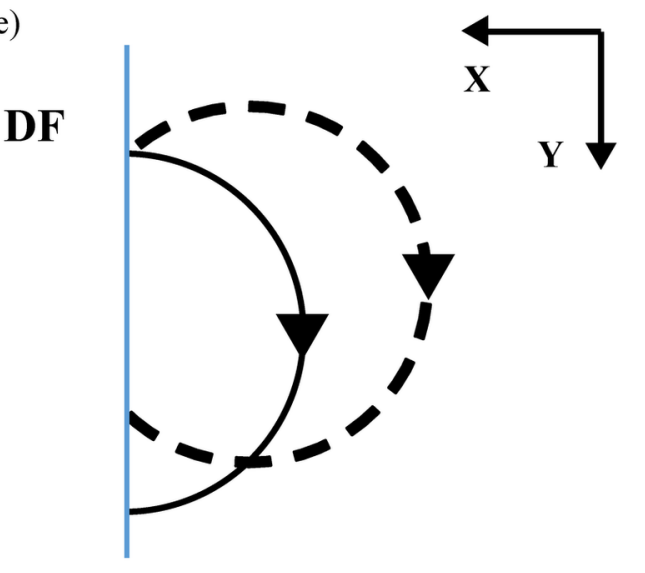

2018JA025689-f06-z-.png

This article is protected by copyright. All rights reserved. 